\title{
Three-Reggeon cuts in QCD amplitudes
}

\author{
V.S. Fadin ${ }^{1,2, *}$ \\ ${ }^{1}$ Budker Institute of Nuclear Physics of SD RAS, 630090 Novosibirsk Russia \\ ${ }^{2}$ Novosibirsk State University, 630090 Novosibirsk Russia
}

\begin{abstract}
One of remarkable properties of QCD is the gluon Reggeization. The Reggeized gluon is the primary Reggeon in QCD; Pomeron and Odderon appear as compound states of the Reggeized gluons. Due to negative signature the Reggeized gluon gives the main contributions to high energy QCD amplitudes in each order of perturbation theory. In the leading and next-to-leading logarithmic approximations these amplitudes are given by the Regge pole contributions. In the next-to-next-to-leading approximation the pole form of the amplitudes is violated by contributions of three-Reggeon cuts. We discuss these contributions to elastic QCD amplitudes.
\end{abstract}

\section{Introduction}

One of remarkable properties of QCD is the Reggeization of all elementary particles in perturbation theory, which is very important for theoretical description of high energy processes.

The gluon Reggeization is especially important because it determines the high energy behaviour of non-decreasing with energy cross sections. In particular, it appears to be the basis of the BFKL (Balitskii-Fadin-Kuraev-Lipatov) equation, which was first derived in nonAbelian theories with spontaneously broken symmetry [1-3] and whose applicability in QCD was then shown [4]. The the amplitudes with gluon quantum numbers in cross-channels are dominant (have the largest $\ln s$ degrees) in each order of perturbation theory, they determine the s-channel discontinuities of amplitudes with the same and all other possible quantum numbers. It is extremely important that both in the leading logarithmic approximation (LLA) [5] and in the next-to-leading one (NLLA) (see [6], [7] and references therein) the amplitudes used in the unitarity relations are determined by the Regge pole contributions and have a simple factorized form (pole Regge form). Due to this, the Reggeization provides a simple derivation of the BFKL equation in the LLA and in the NLLA. The However, the Regge pole contributions are not sufficient in the NNLLA.

For elastic scattering processes $A+B \rightarrow A^{\prime}+B^{\prime}$ in the Regge kinematic region: $s \simeq-u \rightarrow$ $\infty, t$ fixed (i.e. not growing with $s$ ) the Reggeization means that scattering amplitudes with the gluon quantum numbers in the $t$-channel and negative signature (symmetry with respect to $s \leftrightarrow u$ ) are written as

$$
\mathcal{A}_{A B}^{A^{\prime} B^{\prime}}=\Gamma_{A^{\prime} A}^{c}\left[\left(\frac{-s}{-t}\right)^{j(t)}-\left(\frac{s}{-t}\right)^{j(t)}\right] \Gamma_{B^{\prime} B}^{c},
$$

\footnotetext{
*e-mail: fadin@inp.nsk.su
}

Work supported in part by the Ministry of Science and Higher Education of Russian Federation, in part by RFBR, grant 19-02-00690 
where $\Gamma_{P^{\prime} P}^{c}$ are particle-particle-Reggeon (PPR) vertices or scattering vertices ("c" are Reggeon colour indices); $j(t)=1+\omega(t)$ - Reggeon trajectory.

The important property of Regge poles is the factorization of their contributions

$$
\mathcal{A}_{g g}^{g^{\prime} g^{\prime}} \mathcal{A}_{q q}^{q^{\prime} q^{\prime}}=\left(\mathcal{A}_{g q}^{g^{\prime} q^{\prime}}\right)^{2}
$$

The first observation of the pole Regge form (1) was done in [8] at consideration of the highenergy limit of the two-loop amplitudes for $g g, g q$ and $q q$ scattering. The discrepancy appears in non-logarithmic terms, which correspond to the NNLLA.

Using the infrared factorization techniques, consideration of the terms responsible for breaking of the pole Regge form in amplitudes of elastic scattering in QCD was performed by [9]-[11]. In particular, the non-logarithmic terms not satisfying the factorization condition at two-loops were recovered and single-logarithmic terms at three loops violating the pole Regge form were found.

It was natural to expect that the observed violation of the pole Regge form can be explained by existence of the Regge cuts. Indeed, all known cases of breaking of the pole Regge form are now explained by the three-Reggeon cuts [12], [13]. However, the approaches used for determination of the cut contributions in [12] (see although [14], [15]) and [13] are different. Therefore the question requires further investigation. The choice between the approaches can be made based on higher approximations. We discuss here the cut contributions up to four loops.

\section{Two loops}

Our approach is based on consideration of Feynman diagrams. Due to the signature conservation, the cut with negative signature must be the three-Reggeon one. Since our Reggeon is the Reggeized gluon, the three-Reggeon cut first contribute to amplitudes corresponding to the Feynman diagrams with three gluons in the $t$-channels, which differ in permutations $\sigma$ (taking values $a, b, c, d, e, f)$ of the gluon vertices. The amplitudes $\mathcal{A}_{A B}^{A^{\prime} B^{\prime}}$ can be written as the sum over the permutations of products of colour factors and colour-independent matrix elements:

$$
\mathcal{A}_{A B}^{A^{\prime} B^{\prime}}=\sum_{\sigma}\left(C_{A B}^{(0) \sigma}\right)_{\alpha \beta}^{\alpha^{\prime} \beta^{\prime}} M_{A B}^{(0) \sigma}(s, t),
$$

where $\alpha$ and $\beta$ ( $\alpha^{\prime}$ and $\beta^{\prime}$ ) are colour indices of incoming (outgoing) projectile $A$ and target $B$ respectively. We use the same letters for quark and gluon colour indices; it should be remembered, however, that there is no difference between upper and lower indices (running from 1 to $N_{c}^{2}-1$ ) for gluons, whereas for quarks lower and upper indices (running from 1 to $N_{c}$ ) refer to mutually related representations.

The colour factors are

$$
\left(C_{A B}^{(0) \sigma}\right)_{\alpha \beta}^{\alpha^{\prime} \beta^{\prime}}=\left(\mathcal{T}_{A}^{c_{1}} \mathcal{T}_{A}^{c_{2}} \mathcal{T}_{A}^{c_{3}}\right)_{\alpha}^{\alpha^{\prime}}\left(\mathcal{T}_{B}^{c_{1}^{\sigma}} \mathcal{T}_{B}^{c_{2}^{\sigma}} \mathcal{T}_{B}^{c_{3}^{\sigma}}\right)_{\beta}^{\beta^{\prime}},
$$

where $\mathcal{T}^{a}$ are the colour group generators in the corresponding representations, $\left[\mathcal{T}^{a}, \mathcal{T}^{b}\right]=$ $i f_{a b c} \mathcal{T}^{c}$; for all representations, $\left(\mathcal{T}^{a}\right)^{b}{ }_{c}=-i f_{a b c}$ for gluons, $\left(\mathcal{T}^{a}\right)^{\alpha^{\prime}}{ }_{\alpha}=\left(t^{a}\right)^{\alpha^{\prime}}{ }_{\alpha}$ for quarks; $\operatorname{Tr}\left(\mathcal{T}_{A}^{a} \mathcal{T}_{B}^{b}\right)=T_{A} \delta_{A B} \delta_{a b}, \quad T_{q}=1 / 2, \quad T_{g}=N_{c}$. The colour factors can be decomposed into irreducible representations $\mathbf{R}$ of the colour group in the $t$-channel:

$$
\left(C_{A B}^{(0) \sigma}\right)_{\alpha \beta}^{\alpha^{\prime} \beta^{\prime}}=\sum_{\mathbf{R}}\left[\mathcal{P}_{A B}^{\mathbf{R}}\right]_{\alpha \beta}^{\alpha^{\prime} \beta^{\prime}} \mathcal{G}(\mathbf{R})_{A B}^{(0) \sigma},
$$


where

$$
\left[\mathcal{P}_{A B}^{\mathbf{R}}\right]_{\alpha \beta}^{\alpha^{\prime} \beta^{\prime}}=\sum_{n}\left[\mathcal{P}_{A}^{\mathbf{R}, n}\right]_{\alpha}^{\alpha^{\prime}}\left[\mathcal{P}_{B}^{\mathbf{R}, n}\right]_{\beta}^{\beta^{\prime}}
$$

$\hat{\mathcal{P}}^{\mathbf{R}, n}$ is the projection operator on the state $n$ in the representation $\mathbf{R}$ with the normalization

$$
\left[\mathcal{P}_{P}^{\mathbf{R}, n}\right]_{\beta}^{\alpha}\left[\mathcal{P}_{P}^{\mathbf{R}^{\prime}, n^{\prime} *}\right]_{\alpha}^{\beta}=T_{P} \delta_{\mathbf{R}, \mathbf{R}^{\prime}} \delta_{n, n^{\prime}}
$$

so that

$$
\mathcal{G}(\mathbf{R})_{A B}^{(0) \sigma}=\frac{1}{N_{\mathbf{R}} T_{A} T_{B}}\left(\mathcal{T}_{A}^{c_{1}} \mathcal{T}_{A}^{c_{2}} \mathcal{T}_{A}^{c_{3}}\right)_{\alpha}^{\alpha^{\prime}}\left(\mathcal{T}_{B}^{c_{1}^{\sigma}} \mathcal{T}_{B}^{c_{2}^{\sigma}} \mathcal{T}_{B}^{c_{3}^{\sigma}}\right)_{\beta}^{\beta^{\prime}}\left[\mathcal{P}_{A B}^{\mathbf{R} *}\right]_{\alpha^{\prime} \beta^{\prime}}^{\alpha \beta},
$$

$N_{\mathbf{R}}$ is the dimension of the representation $\mathbf{R}$.

In contrast to the Reggeon, which contributes only to amplitudes with the adjoint representation of the colour group (colour octet in QCD) in the $t$-channel, the cut can contribute to various representations.

Possible representations for quark-quark and quark-gluon scattering are only singlet (1) and octet $(\mathbf{8})$, whereas for the gluon-gluon scattering there are also $\mathbf{1 0}, \mathbf{1 0}^{*}$ and 27.

Taking into account Bose statistic for gluons, symmetry of the representations $\mathbf{1}$ and 27, antisymmetry 10 and $10^{*}$ and existence both symmetric $\mathbf{8}_{\mathrm{s}}$ and antisymmetric $\mathbf{8}_{\mathrm{a}}$ representations for them, gives that amplitudes with negative signature there are in the representations besides the Reggeon channel $\mathbf{8}$ amplitudes with negative signature $\mathbf{1}$ for quark-quarkscattering and in the representation 10 and $10^{*}$ for the gluon-gluon scattering.

The important thing is the very existence of amplitudes with negative signature and with the representations of the colour group other than the associated representation. Since such amplitudes could not be given by the Reggeized gluon, their very existence means violation of the pole factorization in the NNLLA.

The projection operators for the octet channel are:

$$
\left[\mathcal{P}_{g g}^{\mathbf{8}_{\mathbf{a}}}\right]_{a^{\prime} b^{\prime}}^{a b}=f_{a a^{\prime} c} f_{b b^{\prime} c}
$$

for gluon-gluon scattering,

$$
\left[\mathcal{P}_{g q}^{\mathbf{8}_{\mathbf{a}}}\right]_{a \beta}^{a^{\prime} \beta^{\prime}}=-i f_{a a^{\prime}}^{c}\left(t^{c}\right)_{\beta}^{\beta^{\prime}}
$$

for gluon-quark scattering, and

$$
\left[\mathcal{P}_{q q}^{\mathbf{8}}\right]_{\alpha \beta}^{\alpha^{\prime} \beta^{\prime}}=\left(t^{c}\right)_{\alpha}^{\alpha^{\prime}}\left(t^{c}\right)_{\beta}^{\beta^{\prime}}
$$

for quark-quark scattering.

The channels 10 and $10^{*}$ exist only for gluon-gluon scattering. The projection operators are

$$
\begin{gathered}
{\left[\mathcal{P}_{g g}^{\mathbf{1 0}}\right]_{a^{\prime} b^{\prime \prime}}^{a b}=\frac{N_{c}}{4}\left(\delta_{a b} \delta_{a^{\prime} b^{\prime}}-\delta_{a b^{\prime}} \delta_{a^{\prime} b}-2 \frac{f_{a a^{\prime} c} f_{b b^{\prime} c}}{N_{C}}+i f_{b a^{\prime} c} d_{b^{\prime} a c}+i d_{b a^{\prime} c} f_{b^{\prime} a c}\right),} \\
{\left[\mathcal{P}_{g g}^{\mathbf{1 0}^{*}}\right]_{a^{\prime} b^{\prime \prime}}^{a b}=\left(\left[\mathcal{P}_{g g}^{\mathbf{1 0}}\right]_{a^{\prime} b^{\prime \prime}}^{a b}\right)^{*} .}
\end{gathered}
$$

And finally, in the negative signature the channel 1 exists only for quark-quark scattering; the projection operators is

$$
\left[\mathcal{P}_{q q}^{\mathbf{1}}\right]_{\alpha \beta}^{\alpha^{\prime} \beta^{\prime}}=\frac{1}{2 N_{c}} \delta_{\alpha}^{\alpha^{\prime}} \delta_{\beta}^{\beta^{\prime}} .
$$

It turns out that for the representations $\mathbf{R}$ different from the Reggeized gluon one the colour coefficients $\mathcal{G}(\mathbf{R})_{A B}^{(0) \sigma}$ do not depend on $\sigma$; they are equal

$$
\mathcal{G}\left(\mathbf{1 0}+\mathbf{1 0}^{*}\right)_{g g}^{(0) \sigma}=-\frac{3}{4} N_{c}, \quad \mathcal{G}(\mathbf{1})_{q q}=\frac{\left(N_{c}^{2}-4\right)\left(N_{c}^{2}-1\right)}{16 N_{c}^{3}},
$$


irrespective of $\sigma$. Therefore the momentum dependent factors for them summed up to the eikonal amplitude

$$
\sum_{\sigma} M_{A B}^{(0) \sigma}(s, t)=A^{e i k}=g^{6} \frac{s}{t}\left(\frac{-4 \pi^{2}}{3}\right) \vec{q}^{2} A_{2}\left(q_{\perp}\right),
$$

where

$$
A_{2}\left(q_{\perp}\right)=\int \frac{d^{2+2 \epsilon} l_{1} d^{2+2 \epsilon} l_{2}}{(2 \pi)^{2(3+2 \epsilon)} \vec{l}_{1}^{2} \vec{l}_{2}^{2}\left(\vec{q}-\vec{l}_{1}-\vec{l}_{2}\right)^{2}} .
$$

Note that the "infrared" $\epsilon$ is used, $\epsilon=(D-4) / 2, D$ is the space-time dimension.

This result is very important, because contribution of the cut must be gauge invariant, whereas $M_{A B}^{(0) \sigma}$ taken separately are gauge dependent.

In the Reggeized gluon channel the colour coefficients $\mathcal{G}(R)_{A B}^{(0) \sigma}$ depend on $\sigma$. However, this dependence has a specific form. Let $\sigma=a(\sigma=f)$ relate to the diagram without $u(s)$ channel cuts. Note that since $M_{A B}^{(0) a}$ and $M_{A B}^{(0) f}$ are connected by the replacement $s \leftrightarrow u$, only the sum $\mathcal{G}\left(\mathbf{8}_{\mathbf{a}}\right)_{A B}^{(0) a}+\mathcal{G}\left(\mathbf{8}_{\mathbf{a}}\right)_{A B}^{(0) f}$ enters in the amplitudes with negative signature. It turns out that

$$
\frac{1}{2}\left[\mathcal{G}\left(\mathbf{8}_{\mathbf{a}}\right)_{A B}^{(0) a}+\mathcal{G}\left(\mathbf{8}_{\mathbf{a}}\right)_{A B}^{(0) f}\right]=\mathcal{G}\left(\mathbf{8}_{\mathbf{a}}\right)_{A B}^{(0)}+\frac{N_{c}^{2}}{8},
$$

whereas for all other diagrams $(\sigma=b, c, d, e) \mathcal{G}\left(\mathbf{8}_{\mathbf{a}}\right)_{A B}^{(0) \sigma}=\mathcal{G}\left(\mathbf{8}_{\mathbf{a}}\right)_{A B}^{(0)}$. The Regge pole factorization requires the equality

$$
\mathcal{G}\left(\mathbf{8}_{\mathbf{a}}\right)_{g g}^{(0) \sigma}+\mathcal{G}\left(\mathbf{8}_{\mathbf{a}}\right)_{q q}^{(0) \sigma}=2 \mathcal{G}\left(\mathbf{8}_{\mathbf{a}}\right)_{g q}^{(0) \sigma}
$$

Since

$$
\mathcal{G}\left(\mathbf{8}_{\mathbf{a}}\right)_{g g}^{(0)}=\frac{3}{2}, \quad \mathcal{G}\left(\mathbf{8}_{\mathbf{a}}\right)_{g q}^{(0)}=\frac{1}{4}, \quad \mathcal{G}\left(\mathbf{8}_{\mathbf{a}}\right)_{q q}^{(0)}=\frac{1}{4}\left(-1+\frac{3}{N_{c}^{2}}\right),
$$

it is evident that the pole factorization is violated.

But it is seen also that the terms violating the pole factorization have $\sigma$-independent colour coefficients, so that momentum factors for them summed up to the eikonal amplitude.

However, the colour factors (19) cannot be fully attributed to the cut contributions. Indeed, separation of the pole and cut contributions is impossible in the two-loop approximation because of the ambiguity of the allocation of the part of the amplitudes violating the factorization: it is always possible to write

$$
\mathcal{G}\left(\mathbf{8}_{\mathbf{a}}\right)_{A B}^{(0)}=\mathcal{G}\left(\mathbf{8}_{\mathbf{a}}\right)_{A B}^{(0) C}+\mathcal{G}\left(\mathbf{8}_{\mathbf{a}}\right)_{A B}^{(0) R}
$$

with $\mathcal{G}\left(\mathbf{8}_{\mathbf{a}}\right)_{A B}^{(0) R}$ satisfying the factorization condition but otherwise arbitrary.

\section{Three loops}

The separation becomes possible in higher loops, due to different energy dependence of the pole and cut contributions. Energy dependence of the pole contribution is determined by the Regge factor of the Reggeized gluon $\exp (\omega(t) \ln s)$, where

$$
\omega(t)=-g^{2} N_{c} \vec{q}^{2} \int \frac{d^{2+2 \epsilon} l}{2(2 \pi)^{(3+2 \epsilon)} \vec{l}^{2}(\vec{q}-\vec{l})^{2}}
$$

is the gluon trajectory, whereas for the three-Reggeon cut it is $\exp (\hat{\mathcal{K}} \ln s)$, where

$$
\hat{\mathcal{K}}=\hat{\omega}_{1}+\hat{\omega}_{2}+\hat{\omega}_{3}+\hat{\mathcal{K}}_{r}(1,2)+\hat{\mathcal{K}}_{r}(1,3)+\hat{\mathcal{K}}_{r}(2,3)
$$


$\hat{\omega}_{i}$ stands for the $i$-th gluon trajectory and $\hat{\mathcal{K}}_{r}(m, n)$ is the real part of the BFKL kernel describing interaction between Reggeons $m$ and $n$. The explicit form of the real part of the kernel describing interaction between two Reggeons with transverse momenta $\vec{l}_{1}$ and $\vec{l}_{2}$ and colour indices $c_{1}$ and $c_{2}$ is

$$
\left[\mathcal{K}_{r}\left(\vec{q}_{1}, \vec{q}_{2} ; \vec{k}\right)\right]_{c_{1} c_{2}}^{c_{1}^{\prime} c_{2}^{\prime}}=-T_{c_{1} c_{1}^{\prime}}^{a} T_{c_{2} c_{2}^{\prime}}^{a} \frac{g^{2}}{(2 \pi)^{D-1}}\left[\frac{\vec{q}_{1}^{2} \vec{q}_{2}^{\prime 2}+\vec{q}_{2}^{2} \vec{q}_{1}^{\prime 2}}{\vec{k}^{2}}-\vec{q}^{2}\right],
$$

where $\vec{q}_{1}+\vec{q}_{2}=\vec{q}_{1}^{\prime}+\vec{q}_{2}^{\prime}=\vec{q}, \quad \vec{q}_{1}-\vec{q}_{1}^{\prime}=\vec{q}_{2}^{\prime}-\vec{q}_{2}=\vec{k}$.

It turns out that the first order colour coefficient, $\mathcal{G}(R)_{A B}^{(n) \sigma}$ is simply proportional to $\mathcal{G}(R)_{A B}^{(0) \sigma}$. As for the trajectory contributions, it is evident. It is true also for the real part contributions, because the colour operator $\sum_{i>j=1}^{3} \hat{T}^{c}(i) \hat{T}^{c}(j)$ acts on the state $\Psi(\mathbf{R})$ such that

$$
\left(\sum_{i=1}^{3} \hat{T}^{c}(i)+\hat{\mathcal{T}}^{c}(\mathbf{R})\right) \Psi(\mathbf{R})=0
$$

where $\hat{\mathcal{T}}^{c}(\mathbf{R})$ is the colour group generator in the representation $R$, due to colour conservation. It gives

$$
\sum_{i>j=1}^{2} \hat{T}^{c}(i) \hat{T}^{c}(j) \Psi(\mathbf{R})=\frac{1}{2}\left(C_{2}(\mathbf{R})-3 C_{2}(\mathbf{8})\right) \Psi(\mathbf{R}),
$$

where $C_{2}(\mathbf{R})$ is the value of the Casimir operator in the representation $R ; C_{2}(\mathbf{8})=N_{c}$.

The calculation of the three-loop corrections [14] shows that the violation of the pole Regge form, analysed in this approximation with the help of the infrared factorization, can be explained by the pole and cut contributions. In other words, the restrictions imposed by the infrared factorization on the parton scattering amplitudes with the adjoint representation of the colour group in the $t$-channel and negative signature can be fulfilled in the NNLLA at two and three loops if besides the Regge pole contribution there is the Regge cut contribution

$$
\begin{gathered}
\mathcal{G}\left(\mathbf{8}_{\mathbf{a}}\right)_{A B}^{(c u t)} g^{6} \frac{s}{t}\left(\frac{-4 \pi^{2}}{3}\right) \vec{q}^{2}\left(A_{2}\left(q_{\perp}\right)+g^{2} N_{c} \ln s\left(\frac{1}{2} A_{3}^{b}\left(q_{\perp}\right)-A_{3}^{c}\left(q_{\perp}\right)\right),\right. \\
A_{3}^{b}\left(q_{\perp}\right)=-\int \frac{d^{2+2 \epsilon} l_{1} d^{2+2 \epsilon} l_{2} d^{2+2 \epsilon} l_{3}}{(2 \pi)^{3(3+2 \epsilon)} \vec{l}_{1}^{2} \vec{l}_{2}^{2} \vec{l}_{3}^{2}\left(\vec{q}-\vec{l}_{1}-\vec{l}_{2}-\vec{l}_{3}\right)^{2}}, \\
A_{3}^{c}\left(q_{\perp}\right)=\int \frac{d^{2+2 \epsilon} l_{1} d^{2+2 \epsilon} l_{2} d^{2+2 \epsilon} l_{3}\left(\vec{q}-\vec{l}_{1}\right)^{2}}{(2 \pi)^{3(3+2 \epsilon)} \vec{l}_{1}^{2} \vec{l}_{2}^{2} \vec{l}_{3}^{2}\left(\vec{q}-\vec{l}_{1}-\vec{l}_{2}\right)^{2}\left(\vec{q}-\vec{l}_{1}-\vec{l}_{3}\right)^{2}} . \\
\mathcal{G}\left(\mathbf{8}_{\mathbf{a}}\right)_{g g}^{(c u t)}=-\frac{3}{2}, \quad \mathcal{G}\left(\mathbf{8}_{\mathbf{a}}\right)_{g q}^{(c u t)}=-\frac{3}{2}, \quad \mathcal{G}\left(\mathbf{8}_{\mathbf{a}}\right)_{q q}^{(c u t)}=\frac{3\left(1-N_{c}^{2}\right)}{4 N_{c}^{2}} .
\end{gathered}
$$

This result is limited to three loops and can not be considered as a proof that in the NNLLA the only singularities in the $J$ plane are the Regge pole and the three-Reggeon cut. Moreover, the explanation of the violation of the pole Regge form given in [13] differs from described above. In this paper, no connection of the three-Reggeon cuts with Feynman diagrams was traced and the colour coefficients $\mathcal{G}(\mathbf{R})_{A B}^{(0) C}$ for the cut contributions

$$
\mathcal{G}(\mathbf{R})_{A B}^{(0) C}=\frac{1}{6 ! N_{\mathbf{R}} T_{A} T_{B}}\left(\mathcal{T}_{A}^{c_{1}} \mathcal{T}_{A}^{c_{2}} \mathcal{T}_{A}^{c_{3}}\right)_{\alpha}^{\alpha^{\prime}}\left(\sum_{\sigma} \mathcal{T}_{B}^{c_{1}^{\sigma}} \mathcal{T}_{B}^{c_{2}^{\sigma}} \mathcal{T}_{B}^{c_{3}^{\sigma}}\right)_{\beta}^{\beta^{\prime}}\left[\mathcal{P}_{A B}^{\mathbf{R} *}\right]_{\alpha^{\prime} \beta^{\prime}}^{\alpha \beta}
$$


were in fact postulated, without any derivation and justification. Since for the representations $\mathbf{R}$ different from the Reggeized gluon one the colour coefficients $\mathcal{G}(\mathbf{R})_{A B}^{(0) \sigma}$ do not depend on $\sigma$ (see (14)), the cut contributions for these representations in both approaches are the same. But it is not so for the adjoint representation, where the colour coefficients $\mathcal{G}(\mathbf{R})_{A B}^{(0) C}$ of [13] turn out to be

$$
\mathcal{G}(\mathbf{R})_{A B}^{(0) C}=\mathcal{G}(\mathbf{R})_{A B}^{(0)}+\frac{N_{c}^{2}}{24},
$$

where $\mathcal{G}(\mathbf{R})_{A B}^{(0)}$ are given by (19), and therefore it is not suppressed at large $N_{c}$, i.e. it exists in the planar $N=4 \mathrm{SYM}$, in contradiction with the common wisdom.

Because of the difference in the colour coefficients for the adjoint representation, the Reggeon-cut mixing is introduced in [13] to explain the violation of the pole factorization, whereas in our approach, the Reggeon-cut mixing is not necessary in the three-loop approximation.

\section{Four loops}

In the four loops there are three types of corrections. The first (simplest) ones come from account of the Regge factors of each of three Reggeons. The second type of the corrections are given by the products of the trajectories and real parts of the BFKL kernel, and the third come from account of Reggeon-Reggeon interactions.

$$
\begin{gathered}
I_{i}=\int \frac{d^{2+2 \epsilon} l_{1} d^{2+2 \epsilon} l_{2} d^{2+2 \epsilon} l_{3}}{(2 \pi)^{3(3+2 \epsilon)} \vec{l}_{1}^{2} \vec{l}_{2}^{2} \vec{l}_{3}^{2}} F_{i} \delta^{3+2 \epsilon}\left(\vec{q}-\vec{l}_{1}-\vec{l}_{2}-\vec{l}_{3}\right), \\
F_{a}=f_{1}\left(\vec{l}_{1}\right) f_{1}\left(\vec{l}_{2}\right), \quad F_{b}=f_{1}\left(\vec{l}_{1}\right) f_{1}\left(\vec{l}_{1}\right), \quad F_{c}=f_{2}\left(\vec{l}_{1}+\vec{l}_{2}\right), \\
F_{d}=f_{1}\left(\vec{l}_{1}+\vec{l}_{2}\right) f_{1}\left(\vec{l}_{1}+\vec{l}_{2}\right), \quad F_{e}=f_{1}\left(\vec{q}-\vec{l}_{1}\right) f_{1}\left(\vec{q}-\vec{l}_{3}\right), \\
f_{1}(\vec{k})=\vec{k}^{2} \int \frac{d^{2+2 \epsilon} l}{(2 \pi)^{(3+2 \epsilon)} \vec{l}^{2}(\vec{l}-\vec{k})^{2}}, \quad f_{2}(\vec{k})=\int \frac{d^{2+2 \epsilon} l f_{1}(\vec{l})}{(2 \pi)^{(3+2 \epsilon)} \vec{l}^{2}(\vec{l}-\vec{k})^{2}} .
\end{gathered}
$$

These integrals enter in the total four-loop correction with different colour factors in the approaches with or without the Reggeon-cut mixing.

Calculation of the colour factors $\mathcal{G}(\mathbf{R})_{A B}^{(2) \sigma}$ is not easy.

Of course, it is trivial for the squared virtual part contribution. The corresponding colour factor is

$$
\mathcal{G}(R)_{A B, V V}^{(2) \sigma}=\frac{\left.N_{c}^{2}\right)}{4} \mathcal{G}(\mathbf{R})_{A B}^{(0) \sigma}
$$

It is not difficult also for the products of the virtual parts due to the property (23). It gives

$$
\mathcal{G}(R)_{A B, V R}^{(2) \sigma}=\frac{N_{c}}{2}\left(C_{2}(\mathbf{R})-3 N_{c}\right) \mathcal{G}(\mathbf{R})_{A B}^{(0) \sigma}
$$

However, it is rather complicated for the squared real part. It contains the matrix elements

$$
\left\langle\Psi_{B}^{\sigma}\left|\sum_{i \neq j=1}^{3} \hat{T}^{c}(i) \hat{T}^{c}(j) \hat{T}^{d}(i) \hat{T}^{d}(j)\right| \Psi_{A}\right\rangle
$$

and

$$
\left\langle\Psi_{B}^{\sigma}\left|\sum_{i \neq j \neq k=1}^{3} \hat{T}^{c}(i) \hat{T}^{c}(j) \hat{T}^{d}(i) \hat{T}^{d}(k)\right| \Psi_{A}\right\rangle
$$


Due to (23) their difference is equal to

$$
\frac{1}{4}\left(C_{2}(\mathbf{R})-3 N_{c}\right)^{2} \mathcal{G}(\mathbf{R})_{A B}^{(0) \sigma},
$$

therefore it is sufficient to obtain the first one.

Rather tedious calculations give for its contribution to $\mathcal{G}\left(\mathbf{8}_{\mathbf{a}}\right)_{A B}^{(s) \sigma}$ to $\mathcal{G}(R)_{A B}^{(2) \sigma}$ :

$$
\begin{gathered}
\mathcal{G}\left(\mathbf{8}_{\mathbf{a}}\right)_{A B}^{(s) b}=\mathcal{G}\left(\mathbf{8}_{\mathbf{a}}\right)_{A B}^{(s) c}=\mathcal{G}\left(\mathbf{8}_{\mathbf{a}}\right)_{A B}^{(s) d}=\mathcal{G}\left(\mathbf{8}_{\mathbf{a}}\right)_{A B}^{(s) e}=\mathcal{G}\left(\mathbf{8}_{\mathbf{a}}\right)_{A B}^{(s)}, \\
\frac{1}{2}\left(\mathcal{G}\left(\mathbf{8}_{\mathbf{a}}\right)_{A B}^{(s) a}+\mathcal{G}\left(\mathbf{8}_{\mathbf{a}}\right)_{A B}^{(s) f}\right)=\mathcal{G}\left(\mathbf{8}_{\mathbf{a}}\right)_{A B}^{(s)}+\left(\frac{N_{c}^{4}}{16}+\frac{3 N_{c}^{2}}{8}\right) .
\end{gathered}
$$

It means that as well as in the two and three loops the terms violating the pole factorization have $\sigma$-independent colour coefficients, which provides their gauge invariance.

In the approach of [13], the four-loop colour coefficients are different from ours. It makes possible to discriminate one of this approaches.

The question of whether the four-loop amplitudes of elastic scattering in QCD are given by the Regge pole and cut contributions, in some approach, can be solved by comparing of the corrections with the results of other calculations. Unfortunately, one can hardly hope that direct calculations will be carried out in the foreseeable future. However, corresponding results can be obtained using the infrared factorization.

\section{Summary}

The gluon Reggeization is the basis of the BFKL approach. The BFKL equation was derived assuming the pole Regge form of amplitudes with gluon quantum numbers in cross channels and negative signature. It is proved now in all orders of perturbation theory that this form is valid both in the leading and in the next-to-leading logarithmic approximations. However, this form is violated in the NNLLA.

The breaking the pole Regge form should have been expected, because it is well known that Regge poles in the complex angular momenta plane generate Regge cuts. Moreover, in amplitudes with positive signature the Regge cuts appear already in the LLA. In particular, the BFKL Pomeron is the two-Reggeon cut in the complex angular momenta plane. But in amplitudes with negative signature Regge cuts must be at least three-Reggeon ones and can appear only in the NNLLA. Therefore it is natural to expect that the observed violation is due to the cut contributions.

It was shown that the observed violation can be explained by the three-Reggeon cuts $[12,13]$. But the assertion that the QCD amplitudes with gluon quantum numbers in crosschannels and negative signature are given in the NNLLA by the contributions of the Regge pole and the three-Reggeon cut is only a hypotheses, and as yet there is no general proof of it, it should be checked in each order of the perturbation theory. Besides this, the approaches used and the explanations given in [12] and [13] are different.

The calculations of the cut contributions presented here aim to prove this hypothesis in four loops and to make choice between two approaches. It can be done comparing their results with the results of other calculations. Unfortunately, direct calculations in that order in the NNLLA do not exist, and there is no hope that they will be done in foreseeable future. But it seems possible to obtain corresponding results using the infrared factorization. Comparison of the results should answer the questions, whether the existence of a pole and a cut is sufficient, with or without mixing. 
It is necessary to understand, however, that fixed order calculations can not prove that the QCD amplitudes with gluon quantum numbers in cross-channels and negative signature are given in the NNLLA by the contributions of the Regge pole and the three-Reggeon cut.

Some idea of the proof, like bootstrap of the gluon Reggeization, but with account of the cut, is required.

\section{References}

[1] V.S. Fadin, E.A. Kuraev, and L.N. Lipatov, Phys. Lett. B 60, 50-52 (1975).

[2] E.A. Kuraev, L.N. Lipatov, and V.S. Fadin, Zh. Eksp. Teor. Fiz. 71, 840-855 (1976).

[3] E.A. Kuraev, L.N. Lipatov, and V.S. Fadin, Zh. Eksp. Teor. Fiz. 72, 377-389 (1977).

[4] I.I. Balitsky, and L.N. Lipatov, Yad. Fiz. 28, 1597-1611 (1978).

[5] Ya.Ya. Balitskii, L.N. Lipatov, and V.S. Fadin, Materials of IV Winter School of LNPI (Leningrad, 1979) 109-149.

[6] Ioffe B.L., Fadin V.S., Lipatov L.N., Quantum chromodynamics: Perturbative and nonperturbative aspects (Cambridge University Press, 2010) 491-504.

[7] Fadin V.S., Kozlov M.G., Reznichenko A.V., Phys. Rev. D. 92, 085044 (2015).

[8] Del Duca V., Glover E.W.N., JHEP. 0110, 035 (2001) .

[9] Del Duca V., Falcioni G., Magnea L., Vernazza L. Phys. Lett. B. 732 , 233 (2014).

[10] Del Duca V., Falcioni G., Magnea L., Vernazza L., PoS RADCOR 2013, 046 (2013).

[11] Del Duca V., Falcioni G., Magnea L., Vernazza L., JHEP 1502, 029 (2015).

[12] Fadin V.S., AIP Conf. Proc. 1819, 060003 (2017).

[13] Caron-Huot S., Gardi E., Vernazza L., JHEP 1706, 016 ( 2017).

[14] V. S. Fadin, PoS DIS 2017, 042 (2018).

[15] V. S. Fadin and L. N. Lipatov, Eur. Phys. J. C 78, 439 (2018). 